\title{
The Relationship of Autism Spectrum Disorders and Celiac Disease and Gluten-free Diet
}

\author{
Anna Szaflarska-Poplawska* \\ Department of Pediatric Endoscopy and Gastrointestinal Function Testing, Ludwik Rydygier Collegium Medicum in Bydgoszcz, \\ Nicolaus Copernicus University in Torun, Poland \\ *Corresponding author: aszaflarska@wp.pl
}

Received August 14, 2015; Revised September 13, 2015; Accepted September 15, 2015

\begin{abstract}
Children with autistic disorders suffer from various gastrological abnormalities more frequently compared to the overall population. Coincidence of autistic disorders and celiac disease is probably a concurrency of two common diseases. However, in order to assess the connection between autistic disorders and non-celiac gluten sensitivity it is necessary to carry out further studies. Gluten-free diet should not be applied in all patients with autistic disorders, but there probably is a group of patients with the diet-related autism phenotype, who can benefit from dietary therapy.
\end{abstract}

Keywords: celiac disease, gluten-free diet, intestinal permeability, autism spectrum disorders

Cite This Article: Anna Szaflarska-Popławska, “The Relationship of Autism Spectrum Disorders and Celiac Disease and Gluten-free Diet.” International Journal of Celiac Disease, vol. 3, no. 4 (2015): 132-135. doi: 10.12691/ijcd-3-4-1.

\section{Introduction}

Autism is a neurodevelopmental disorder, which is clinically manifested by qualitative disturbances of social interaction, verbal and non-verbal communication, and occurrence of limited, repeating stereotypical patterns of activity, behavior and interests. According to the classification proposed in 2013, autism, along with all other previously identified disease entities, is included in a wider category referred to as autism spectrum disorders (ASD) [1,2]. During the last 20 years the prevalence of ASD has increased by more than 10 times [3]. The current prevalence of autistic disorders in the overall population of the United States is estimated at 1 in 88 children. Autism is most frequently diagnosed in early childhood, more often in boys than girls $(4: 1)$ [1,2].

Many studies have indicated an increased incidence of gastrointestinal symptoms in autism spectrum disorders. Moreover, cases of association between autism spectrum disorders and celiac disease were reported. It has been also suggested that dietary intervention, specifically the removal of gluten-containing food from diet may impact on the presentation on autism spectrum disorders. However, the results of studies are conflicting and not conclusive. The aim of this review was to estimate the frequency of gastrointestinal symptoms in autism spectrum disorders and examine the association between celiac disease and autism spectrum disorders. Additionally, a review of the medical literature related to the effectiveness of the gluten-free (and casein-free) elimination diet on autism spectrum disorders was conducted.

\section{The Frequency of Gastrointestinal Symptoms in Autism Spectrum Disorders}

The pathogenesis of autistic disorders is still poorly understood and the commonly used psychological, pedagogic and social management does not lead to recovery [4]. It has been suggested that the pathogenesis involves the so-called "leaky gut" (impaired intestinal permeability). However this hypothesis was not confirmed in all studies and it does not pertain to all patients. In the studies by de Magistris et al., abnormal intestinal permeability measured with use of lactulose and mannnitol test was observed in $36.7 \%$ of patients with autism, $21.2 \%$ of their relatives and in only $4.8 \%$ of healthy subjects [5]. In a different study carried out in the same centre, increased intestinal permeability was confirmed in $25.6 \%$ of patients with autism and $2.3 \%$ of healthy children [4]. Horvath et al. showed increased intestinal permeability with use of the same method in as much as $76 \%$ of children with autism [6].

On the contrary to the authors of the previously quoted studies, Dalton et al. did not find any differences in intestinal permeability between children with autism, compared to children with special educational needs but no autism. In two children, one in each of the investigated groups, intestinal permeability was significantly increased, but it was caused by other conditions (celiac disease, status post partial bowel resection) [7]. Similarly, Robertson et al. did not confirm abnormal intestinal permeability in children with autism and gastrological symptoms [8]. 
Increased intestinal permeability in some patients with autistic disorders allows products of food protein digestion to transfer to the systemic circulation, from where they can pass through the blood-brain barrier, reach the central nervous system and affect child's behavior. High molecular weight proteins, which are suspected of having effect on the functioning of the central nervous system include gliadinomorphin-7 (YPQPQPF; GM7) produced in the process of enzymatic digestion of wheat, $\alpha$-gliadin $\mathrm{h} \beta$-casomorphin-7 (YPFVEPL; hBCM7) and b $\beta$ casomorphin-7 (YPFPGPL; bBCM7) produced in the process of hydrolysis of milk $\beta$-casein (human and cow's, respectively). Presence of 2-3 proline residues makes these proteins resistant to further proteolysis. They are ascribed to the group of exogenous opioids called exorphins. The fact that they are similar to morphine and can activate opioid receptors became the basis of the opioid peptide theory proposed by Panksepp in 1979. Activation of $\mu$-opioid receptors induces changes in the EAAT3 expression (excitatory amino acid transporters), which then affects cysteine uptake by mature nervous cells and epithelial cells of the gastrointestinal tract, especially in the distal part of the ileum. In consequence, there is a decrease in the bioavailability of S-adenosylmethionine (SAM), which is a donor of methyl groups in over 200 various methylation reactions. This leads to disturbances in DNA methylation and gene transcription [10].

The presuppositions of the opioid theory in the pathogenesis of autistic disorders are challenged because with use of modern diagnostic methods (LC-MS/MS, Mass Spectrometry coupled with high-performance Liquid Chromatography) it was not possible to detect peptide metabolites of opioid activity in the urine of patients with autism [11]. Moreover, the affinity of exogenous opioids to opioid receptors is low, and in food products there are also peptides of antagonistic action to these receptors [2]. According to Julio-Pieper et al., the data confirming abnormal intestinal permeability in patients with various diseases of the central nervous system, including autism, are limited and the effect on affect neurological and behavioral disturbances by affecting the intestinal barrier requires further studies [12].

An attempt to relate autistic disorders with food elements (especially gluten) is at least partially caused by the high frequency of gastrological symptoms that has been for years reported by many authors. The frequency is by various publications estimated at $22-70 \%$ of all patients with autistic disorders [13].

According to the questionnaire carried out among parents of 412 autistic children by Horvath et al., as much as $84.1 \%$ of subjects and $31.2 \%$ of their healthy siblings presented with gastrological symptoms. They most frequently included: discomfort in the abdominal cavity, flatulence and passing gas and disturbances of the number and/or consistency of the stools [14]. Campbell et al. confirmed occurrence of gastrointestinal ailments in 43\% of children with autism and only $4 \%$ of their healthy siblings [15]. According to these authors, the data can be understated due to difficulties with verbal and non-verbal communication, being part of the underlying disease $[3,14]$. In a study by Wang et al., in children with autistic disorders (especially the full autism form) parents significantly more often observed constipation and chronic diarrhea, compared to the healthy siblings. Moreover, the more severe the form of the autistic disorder was, the more prevalent were the gastrological symptoms [16]. According to Pang et al., constipation in children with autism occurs not only more frequently than in the overall population, but also occurs earlier and lasts longer [17]. In the study by Badalyan et al., children with autistic disorders more frequently than their healthy siblings complained about constipation (30\% vs 4\%), stool incontinence ( $22 \%$ vs $2 \%$ ), more frequently had atypical food preferences (57\% vs 5\%) and more frequently refused to eat new types of food (48\% vs 8\%) [18]. Food selectivity in children with autistic disorders was confirmed by Beighley et al. as well [19]. Ibrahim SH et al. showed that constipation and food selectivity were more frequent in children with autistic disorders than in the control group (33.9\% vs $17.6 \%$ and $24.5 \%$ vs $16.1 \%$, respectively). These symptoms are frequently associated with neurobehavioral disturbances, not the primarily organic gastrological condition. Other gastrointestinal symptoms (diarrhea, vomiting, gastroesophageal reflux, discomfort or flatulence) were equally frequent in both investigated groups [20]. According to parents of children studied in 2013 by Chandler et al., $46.5 \%$ of children with autistic disorders, $29.2 \%$ of children with special educational needs and $21.8 \%$ of normally developing children presented with at least one gastrological symptom in their life. Gastrological symptoms did not correlate with intellectual aptitude, severity of autism and dietary limitations [13].

Literature provides single publications, which contradict more frequent occurrence of gastrological symptoms in children with autistic disorders. Black et al. confirmed occurrence of gastrological diseases in only $9 \%$ of children with autism and the same percentage of children without autism [21]. Similarly, Smith et al. did not observe any statistically significant differences in the prevalence of constipation, diarrhea and flatulence in children with autism, compared to healthy children [22]. In a study by Chandler et al., constipations occurred in only $10 \%$ of children with autistic disorders, which means they were as frequent as in the overall population [13].

Authors also point to the possibility of occurrence of gastrointestinal pathologies in children with autistic disorders. In 2005 Wakefield et al. confirmed a significantly higher occurrence of lymphatic follicle hyperplasia in the ileum $(\mathrm{p}<0.0001)$ and the colon $(\mathrm{p}=0.0003)$, compared to the overall population. Lesions of this type in the ileum were found in $90 \%$ of children with autistic disorders, were more severe than in healthy children and did not depend on the type of dietary restrictions applied [23]. Chandler et al. frequently observed macroscopic changes within the upper section of the digestive tract of autistic children. These included reflux esophagitis (69.4\% of children), chronic gastritis (41.6\% of children) or duodenitis (66.6\% of children) and shortening of intestinal villi (5.5\% of children) [14].

\section{The Association between celiac Disease and Autism Spectrum Disorders}

Speculations over the connection of behavioral disturbances with gluten sensitivity date from the 50s of the $20^{\text {th }}$ century. Initially, they were mainly ascribed to 
celiac disease. This was due to the fact that celiac disease was frequently diagnosed in patients with neurological symptoms such as gluten-dependent ataxia, epilepsy, peripheral polyneuropathy, dementia or depression [24]. Neurological symptoms can occur independently or can be accompanied by gastrological symptoms [1].

Studies on the coincidence of autism and celiac disease are scarce. In a small group of 11 children with autism none was found to have serological markers for celiac disease. Moreover, none of the 120 children with celiac disease had behavioral disturbances confirmed, which are typical for autism [25]. Similarly, in a study by Batista et al., including 147 patients with autistic disorders, did not show higher frequency of gluten-dependent diseases (celiac disease and non-celiac gluten sensitivity), compared to the overall population. Similarly, in a group of 211 patients with histopathologically confirmed celiac disease they did not found higher prevalence of autistic disorders than in the overall population [3]. In a study by Chandler et al. only 1 in 132 children with autistic disorders presented with celiac disease [13]. None of the 36 patients examined by Horvath et al. presented with microscopic lesions in the mucosa of the small intestine that are typical for celiac disease [14]. De Magistris et al. found antibodies specific for celiac disease in 2 out of 162 children with autistic disorders [4]. In a study by Lau et al., involving a total number of 140 children, none of the 37 subjects with autism presented with serous celiac-specific tTG-IgA (IgA class antibodies against tissue transglutaminase) and DPG-IgA (IgA class antibodies against deamidated gliadin peptide). However, children with autism were found to have AGA-IgG antibodies more frequently than their healthy peers $(24.2 \%$ vs $5.3 \%$; $\mathrm{p}<0.01$ ). Higher concentration of these antibodies more frequently pertained to autistic children with gastrological symptoms [26]. Based on the data obtained from the Swedish registry of patients with celiac disease it can be observed that earlier diagnosis of autistic disorder was not associated with histopathologically confirmed celiac disease (OR 0.93; 95\%CI 0.51-1.68) and enteritis (OR 1.03; 95\%CI 0.40-2.64), but was associated with over 5time higher risk of abnormal serological tests specific for celiac disease with no inflammatory changes in the small intestine [27].

However, there are studies which indicate that higher coincidence of celiac disease and autistic disorders is possible. Such conclusions were drawn by Barcia et al., who showed that in a group of children with autistic disorders, celiac disease was more than 3-times more frequent than in the overall population (1:106 vs 1:30, $\mathrm{p}=0.014)$. After 6 months of a gluten-free diet they achieved some improvement as regards gastrological symptoms in patients who suffered from them, but no behavioral improvement was observed [28]. Based on a questionnaire, Valicenti-McDermott et al. observed that in a group of 100 children with autistic disorders, patients with speech regression more frequently had positive family history of celiac disease and non-specific inflammatory diseases of the intestine than children with no speech regression ( $24 \%$ vs $0 \%$; $p=0.001$ ) [29].

According to the recommendations of the American Academy of Pediatrics, every child with an autistic disorder and gastrointestinal symptoms should be tested for celiac disease with an assessment of the total concentration of tTG-IgA antibodies with or without antiendomysial antibodies. If gluten-free diet is applied without diagnostic examinations, the patient should undergo a gluten challenge test. Genetical tests for haplotypes HLA-DQ2 and HLA-DQ8 can be useful in exclusion of celiac disease (if the haplotypes mentioned above are not detected) [30].

It is currently suggested that most patients with glutendependent disorder do not suffer from celiac disease but a non-celiac gluten sensitivity. In the majority of patients with non-celiac gluten sensitivity IgG class antibodies against native gliadin (AGA-IgG) can be found. Therefore, the finding of isolated AGA-IgG positivity seems to be the most valuable serological marker of non-celiac gluten sensitivity. In the study by de Magistris et al., AGA-IgG and DPG-IgG occurred in children with autistic disorders more frequently than in the healthy population. The concentration of these antibodies was lower in children who were ill but using the gluten-free diet [4]. In a group of 21 children with autism spectrum disorders 1 patient was diagnosed as having histopatologically confirmed celiac disease and next In autism 7 patients were AGAIgG antibody positive (unpublished personal experience).

The speculations associated with the opioid theory resulted in numerous attempts of application of elimination diets (including gluten-free diet), usually joined with casein-free diet - in order to improve the disturbances of behavior and communication observed in children with autism. Data from the literature indicates that $20-70 \%$ of children with autistic disorders make an attempt to apply gluten-free and casein-free diet [2]. Unfortunately, most studies evaluating the effectiveness of such a diet in children with autistic disorders are of low value. This is mainly due to the fact that there is usually no control group, the studied groups are small and the age heterogeneity of the analyzed group is large. Also there is lack of clear inclusion and exclusion criteria. Among all these studies, one publication of 2000 deserved special attention. It included a group of 150 children aged 3.5-16 years, in whom gluten-free diet resulted in a clinical improvement as regards symptoms of autism [31]. In a randomized clinical trial involving 72 children aged 4-10 years, suffering from autistic disorders, Whiteley et al. showed a significant clinical improvement after application of milk-free and gluten-free diet. However, according to the authors, effect of other, diet-independent, factors cannot be ruled out [32]. On the other hand, in a retrospective, randomized, double-blind study of 13 children aged 2-6 years, that was published in 2007, Seung et al., did not observe any differences in verbal and non-verbal communication of subjects on an elimination and general diet [33]. Similarly, in a randomized, doubleblind study published in 2010, Hyman et al. did not confirm any differences in the behavior of 30 children aged 30-45 months on an 18-month-long gluten-free, casein-free and general diet [34]. The caregivers of children enrolled in a blinded study by Elder et al., in which a 3-month-long gluten- and casein-free diet was applied interchangeably with a 3-month-long general diet, did not observe any differences in the behavior and development of the children, regardless of the type of diet that was applied [35].

In 2008, Millward et al. made an attempt to perform a meta-analysis assessing the effectiveness of gluten-free 
and/or casein-free diet on the improvement of cognitive, social and behavioral functions in children with autism. Unfortunately, they have identified only 2 studies fulfilling the inclusion criteria, based on which they could not formulate any definite conclusions [36]. Similar conclusions were made by authors of later meta-analyses, who concluded that the data confirming the effectiveness of elimination diet in autism is limited and of little scientific value. Gluten-free and milk-free diet should be applied in children with autistic diseases if gluten/milk intolerance or food allergy is diagnosed [37].

\section{Conclusions}

Children with autistic disorders suffer from various gastrological abnormalities more frequently compared to the overall population. Coincidence of autistic disorders and celiac disease is probably a concurrency of two common diseases. However, in order to assess the connection between autistic disorders and non-celiac gluten sensitivity it is necessary to carry out further studies. Gluten-free diet should not be applied in all patients with autistic disorders, but there probably is a group of patients with the diet-related autism phenotype, who can benefit from dietary therapy.

\section{References}

[1] Buie T. The relationship of autism and gluten. Clin Ther 2013; 35: 578-583.

[2] Mari-Bauset S, Zazpe I, Mari-Sanchis A et al. Evidence of the gluten-free and casein-free diet in autism spectrum disorders: a systematic review. J Child Neurol 2014; 29: 1718-1727.

[3] Batista IC, Gandolfi L, Nobrega YKM et al. Autism spectrum disorder and celiac disease: no evidence for a link. Arq Neuropsiquiatr 2012; 70; 28-33.

[4] de Magistris L, Picardi A, Siniscalco D et al. Antibodies against Food Antigens in Patients with Autistic Spectrum Disorders. BioMed Res Int 2013; 729349.

[5] de Magistris L, Familiari V, Pascotto A et al. Alterations of the intestinal barier in patients with autism spectrum disorders and in their first-degree relatives. J Pediatr Gastroenterol Nutr 2010, 51: 418-424.

[6] Horvath K, Zielke H, Collins J et al. Secretin improves intestinal permeability in autistic children. J Pediatr Gastroenterol Nutr 2000; 31: S30-S31.

[7] Dalton N, Chandler S, Turner Ch et al. Gut permeability in autism spectrum disorders. Autism Research 2014; 7: 305-313.

[8] Robertson MA, Sigalet DL, Holst JJ et. Intestinal permeability and glucagon-like peptide 2 in children with autism: a controlled pilot study. J Autism Dev Disord 2008; 38: 1066-1071.

[9] Panksepp J. A neurochemical theory of autism. Trends in Neurosciences 1979; 2: 174-177.

[10] Trivedi MS, Shah JS, Al.-Mughairy S et al. Food-derived opioid peptides inhibit cysteinę uptake with redox and epigenetic consequences. J Nutr Biochem 2014; 25: 1011-1018.

[11] Dettmer K, Hanna D, Whetstone P et al. Autism and urinary exogenous neuropeptides: development of an on-line SPE-HPLCtandem mass spectrometry method to test the opioid excess theory. Anal Bioanal Chem 2007; 388: 1643-1651.

[12] Julio-Pieper M, Bravo JA, Aliaga E, Gotteland M. Review article; intestinal barier dysfunction and central nervous system disorders - a controversial association. Aliment Pharmacol Ther 2014; 40: 1187-1201.

[13] Chandler S, Rathwell IC, Charman T et al. Parent-reported gastrointestinal symptoms in children with autism spectrum disorders. J Autism Dev Disord 2013; 43: 2737-2747.

[14] Horvath K, Perman JA. Autism and gastrointestinal symptoms. Curr Gastroenterol rep 2002; 4: 251-258.
[15] Campbell DB, Buie TM, Winter $\mathrm{H}$ et al. Distnict genetic risk based on association of MET in families with co-occurring autism and gastrointestinal conditions. Pediatrics 2009; 123: 1018-1024.

[16] Wang LW, Tancredi DJ, Thomas DW. The prevalence of gastrointestinal problems in children across the United States with autism spectrum disorders from families with multiple affected members. J Dev Behav Pediatr 2011; 32: 351-360.

[17] Pang KH, Croaker GD. Constipation in children with autism and autistic spectrum disorder. Pediatr Surg Int 2011; 27; 353-358.

[18] Badalyan V, Schwartz RH. Feeding problems and GI dysfunction in children with Asperger Syndrome or pervasive developmental disorders not otherwise specificied; comparison with their siblings. Open J Ped 2011; 1: 51-63.

[19] Ibrahim SH, Voigt RG, Katusic SK et al. Incidence of gastrointestinal symptoms in children with autism: a populationbased study. Pediatrics 2009; 124: 680-686.

[20] Beighley JS, Matson JL, Rieske RD, Adams HL. Food selectivity in children with and without an autism spectrum disorders: investigation of diagnosis and age. Res Dev Disabil 2013; 34: 3497-3503.

[21] Black C, Kaye JA, Jick H. Relation of childhood gastrointestinal disorders to autism: nested case-control study using data from the UK General Practice Research Database. BMJ 2002; 325: 419-421.

[22] Smith RA, Farnworth H, Wright B, Allgar V. Are there more bowel symptoms in children with autism compared to normal children and children with other developmental and neurological disorders? Autism 2009; 13; 343-355.

[23] Wakefield AJ, Ashwood P, Limb K, Anthony A. The significance of ileo-colonic lymphoid nodular hyperplasia in children with autistic disorders. Eur J Gastroenterol Hepatol 2005; 17: 827-836.

[24] Lionetti E, Francavilla R, Pavone P et al. The neurology of coeliac disease in childhood: what is the evidence? A systematic review and meta-analysis. Dev med. Child Neurol 2010; 52: 700-707.

[25] Pavone L, Fiumara A, Bottaro G et al. Autism and celiac disease: failure to validate the hypothesis that a link might exist. Biol Psychiatry 1997; 42: 72-75.

[26] Lau NM, Green PHR, Taylor AK et al. Markers of celiac disease and gluten sensitivity in children with autism. PLOS One 2013; 8(6): e66155.

[27] Ludvigsson JF, Reichenberg A, Hultman CM, Murray JA. A nationwide study of the association between celiac disease and the risk of autisitic spectrum disorders. JAMA Psyciatry 2013; 70: 1224-1230.

[28] Barcia G, Posar A, Santucci M, Parmeggiani A. Autism and coeliac disease. J Autism Dev Disord 2008; 38: 407-408.

[29] Valicenti-McDermott MD, Vicar K, Cohen HJ et al. Gastrointestinal symptoms in children with an autism spectrum disorders and language regression. Pediatr Neurol 2008; 39: 392398.

[30] Buie T, Fuchs GJ, Furuta GT et al. Recommendations for evaluation and treatment of common gastrointestinal problems in children with ASDs. Pediatrics 2010; 125: S19-S29.

[31] Cade R, Privette M, Fregly $M$ et al. Autism and schizophrenia: intestinal disorders. Nutr Neurosci 2000; 3: 57-72.

[32] Whiteley P, Haracopos D, Knivsberg AM et al. The ScanBrit randomised, controlled, single-blind study of a gluten- and caseinfree dietary intervention for children with autism spectrum disorders. Nutr Neurosci 2010; 13; 87-100.

[33] Seung HK, Rogalski Y, Shankar M, Elder J. The gluten-andcasein free diet and autism: communication outcomes from a preliminary double-blind, clinical trial. J Med. Speech Lang Pathol 2007; 15: 337-345.

[34] Hyman S, Steward P, Smith P et al. The gluten free and casein free (GFCF) diet; a double blind, placebo controlled challenge study. Paper presented at the International Meeting for Autism Research, Philadelphia, PA 2010.

[35] Elder JH, Shankar M, Shuster J et al. The gluten-free, casein-free diet in autism: results of a preliminary double blind clinical trial. J Autism Dev Dis 2006; 36: 413-420.

[36] Milllward C, Ferriter M, Calver S et al. Gluten- and casein-free diets for autistic spectrum disorders. Cochrane database Syst Rev 2008; 16: CD003498.

[37] Mulloy A, Lang R, O’Reilly M et al. Gluten-free and casein-free diets in the treatment of autism spectrum disorders: a systematic review. Res Autism Spectr Disord 2011; 5: 86-88. 